\section{SAT0436 \\ PATIENTS WITH PSORIATIC ARTHRITIS WHO ACHIEVE THE MDA RESPONSE SHOW LESS SUBCLINICAL ATHEROSCLEROSIS THAN THOSE IN DAPSA REMISSION}

J. A. Lorenzo Martín ${ }^{1}$, E. Pardo Campo ${ }^{2}$, L. C. Charca Benavente ${ }^{2}$, M. Pino Martínez ${ }^{2}$, S. Alonso Castro ${ }^{2}$, S. Fernández ${ }^{2}$, L. M. Arboleya Rodríguez ${ }^{2}$, M. Alperi-López ${ }^{2}$, R. Queiró Silva ${ }^{2} .{ }^{1}$ Hospital Universitario de Burgos, Burgos, Spain; ${ }^{2}$ Hospital Universitario Central de Asturias, Rheumatology, OVIEDO, Spain

Background: Although the MDA response and DAPSA remission are treatment objectives proposed by EULAR for a proper management of psoriatic arthritis (PsA), there is no clear consensus on which of the two is the most advisable in clinical practice. Some studies suggest that patients who reach a sustained MDA have less subclinical atherosclerosis, but whether the same applies to DAPSA remission is unknown at present.

Objectives: To compare the frequency of subclinical atherosclerosis in patients with PsA that reach the MDA response versus those who achieve DAPSA remission. Methods: One hundred-forty consecutive patients with PSA (CASPAR criteria) treated with biological and non-biological systemic agents were included. SCORE risk charts were used to estimate cardiovascular risk (CVR). The presence of plaques and / or carotid intima-media thickness (cIMT) $>0.9 \mathrm{~mm}$ in carotid ultrasound defined subclinical atherosclerosis. These findings were analyzed in patients in MDA and in those in DAPSA remission.

Results: According to the SCORE tables, $42.8 \%, 35.7 \%$ and $21.5 \%$, had low, moderate and high-very high CVR, respectively. There was a linear association between cIMT values and the SCORE risk categories $(p<0.0005)$. The best cutoff point to define a high CVR (by SCORE plus carotid plaques) corresponded to a cIMT $>0.63 \mathrm{~mm}$, with an area under the ROC curve of $0.75(0.66-0.82)$, sensitivity $85.7 \%$, specificity $56.1 \%$ (Figure 1). Ninety-seven of the 140 patients $(69.3 \%)$ were in MDA situation, while $60(42.8 \%)$ were in DAPSA remission. The average value of the cIMT was $0.64 \pm 0.12 \mathrm{~mm}$. One in 4 patient had atheroma plaques, while $19(13.6 \%)$ had a cIMT $>0.9 \mathrm{~mm}$. There were no differences in CIMT values of patients with $(0.66 \pm 0.12 \mathrm{~mm})$ and without $(0.63 \pm 0.12 \mathrm{~mm})$ DAPSA remission. An identical percentage (25\%) of patients with and without DAPSA remission had carotid atheroma plaques. On the other hand, there were differences between patients with $(6.2 \%)$ and without (30.2\%) MDA in terms of cIMT values $>0.9 \mathrm{~mm}, \mathrm{p}<0.05 .32 .6 \%$ of non-MDA and $23.7 \%$ of MDA patients had carotid plaques, $\mathrm{p}$ NS.

Conclusion: Patients who achieve an MDA response show less subclinical atherosclerosis than those in DAPSA remission. These findings suggest that the MDA response better discriminates the presence of subclinical atherosclerosis, and therefore, it could be a more complete therapeutic target than DAPSA remission.

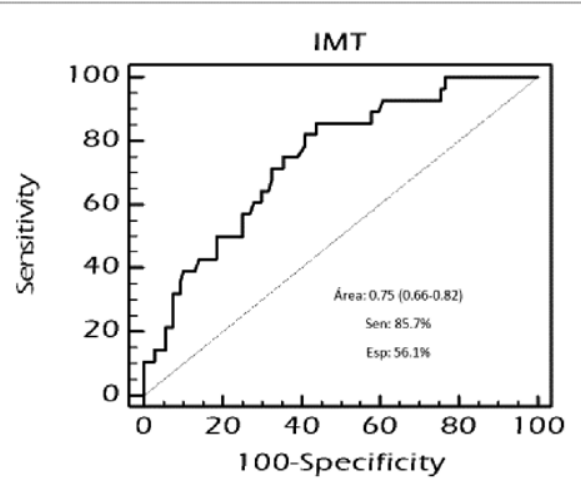

Disclosure of Interests: None declared

DOI: 10.1136/annrheumdis-2020-eular.5256

\section{SAT0437 GENDER DIFFERENCES IN PSA OUTCOME PARAMETERS AND THEIR CORRELATION WITH SKIN INVOLVEMENT: A CROSS-SECTIONAL ANALYSIS OF RABBIT-SPA PATIENTS}

A. Regierer ${ }^{1}, A$. Wei $^{1}$, F. Behrens ${ }^{2}$, M. Feuchtenberger ${ }^{3}$, G. Schett ${ }^{4,5}$, X. Baraliakos ${ }^{6,7}$, A. Zink ${ }^{1,8}$, A. Strangfeld ${ }^{1}{ }^{1}$ Deutsches RheumaForschungszentrum (DRFZ), Epidemiology, Berlin, Germany; ${ }^{2}$ Goethe University Frankfurt, Rheumatology, Frankfurt, Germany; ${ }^{3}$ MED | BAYERN OST MVZ GmbH, Burghausen, Germany; ${ }^{4}$ Universitätsklinikum Erlangen, Rheumatology and Immunology, Erlangen, Germany; ${ }^{5}$ University of Erlangen-Nuremberg, Erlangen, Germany; ${ }^{6}$ Rheumazentrum Ruhrgebiet,
Herne, Germany; ${ }^{7}$ Ruhr-Universität Bochum, Bochum, Germany; ${ }^{8}$ CharitéUniversitätsmedizin Berlin, Berlin, Germany

Background: PsA is a complex disease characterised by a heterogeneous pattern including different clinical symptoms of musculoskeletal (MSK) inflammation like arthritis, enthesitis, dactylitis and axial involvement as well as skin and nail involvement. There are differences in these disease patterns between female and male patients which need to be taken into account.

Objectives: To assess the differences between female and male PsA patients in the extent of psoriatic skin (body surface area, BSA) and joint (tender joint count 68, TJC68; swollen joint count 66, SJC66;) disease, composite scores (disease activity in PSA, DAPSA; disease activity score 28, DAS28), and patient-reported outcomes (PROs) and to correlate the extent of skin disease with PROs.

Methods: RABBIT-SpA is a prospective longitudinal cohort study including PsA patients enrolled at start of a new csDMARD, bDMARD or tsDMARD. Gender specific differences in outcome parameters are compared using Wilcoxon resp. t-test at baseline visit. Spearman correlation coefficient was calculated to analyse the relationship between BSA and outcome parameters.

Results: 722 PsA patients were included in this analysis. Women were slightly older ( 52 vs $50 \mathrm{yrs}$ ), had longer disease duration (7 vs $5.8 \mathrm{yrs}$ ), more comorbidities and were more often obese. Men had a significantly higher skin involvement than women measured by BSA and physician skin assessment (physSk, table 1). Women had significantly higher joint involvement as measured by TJC68, DAPSA, DAS28, and patient muskuloskeletal assessment (patMSK). Impact of disease as measured by PSAID and patient global assessment (patGA) was more severe in women than in men and also physical function (HAQ) was lower in women than in men (table 1). Despite the higher skin involvement in men, the DLQI was equally high in women and men with more than $50 \%$ of patients in reduced quality of life state (table 1 ).

Table 1.

\begin{tabular}{lcc}
\hline Parameter & Female & Male \\
& $\mathrm{N}=424$ & $\mathrm{~N}=298$ \\
\hline SJC66 & $3.4(5)$ & $2.7(3.8)$ \\
TJC68 & $7.9(8.7)$ & $5.7(7)$ \\
BSA (0-100) & $6.6(13)$ & $10.8(16.5)$ \\
physGA (NRS 0-10) & $5.3(1.9)$ & $5.2(2)$ \\
physSk (NRS 0-10) & $3.1(2.7)$ & $3.7(2.6)$ \\
physMSK (NRS 0-10) & $5.2(2.1)$ & $4.9(2.3)$ \\
DAPSA & $24.2(13.8)$ & $20.1(12.3)$ \\
DAS28-CRP & $3.7(1.2)$ & $3.4(1.2)$ \\
patGA (NRS 0-10) & $5.5(2.1)$ & $4.9(2)$ \\
patSk (NRS 0-10) & $3.9(3.1)$ & $3.9(2.8)$ \\
patMSK (NRS 0-10) & $5.8(2.2)$ & $5(2.5)$ \\
DLQI (0-30) & $6.1(6.8)$ & $5.3(5.7)$ \\
HAQ (0-3) & $1.1(0.6)$ & $0.7(0.6)$ \\
PSAID (0-10) & $4.8(2.3)$ & $3.9(2.2)$ \\
\hline
\end{tabular}

Results are presented as mean \pm SD.

BSA was not correlated with SJC66 or TJC68, DAPSA, DAS28, phys MSK patGA and patMSK neither in men nor in women. BSA was however positive correlated with DLQI, patSk, and physSK and slightly with physGA in both genders. The PSAID is correlated to BSA in women only.

Conclusion: Women and men show differences regarding many PsA criteria. Men have a more severe skin involvement, while women have higher burden of joint involvement. In addition in the patient reported parameters women show significantly higher values than men except for the skin specific parameters. Notably, although skin involvement is not correlated with most PsA activity parameters, around $50 \%$ of patients in specialised rheumatologic care are negatively affected in their quality of life by psoriatic skin disease. Therapeutic decisions need to take into account the complexity of the patients' symptoms as well as gender differences.

Acknowledgments: RABBIT-SpA is supported by a joint, unconditional grant from AbbVie, Amgen, Janssen-Cilag, Lilly, MSD, Mylan, Novartis, Pfizer, and UCB. Disclosure of Interests: Anne Regierer Speakers bureau: Novartis, Celgene, Janssen-Cilag, Anja Weiß: None declared, Frank Behrens Grant/research support from: Pfizer, Janssen, Chugai, Celgene, Lilly and Roche, Consultant of: Pfizer, AbbVie, Sanofi, Lilly, Novartis, Genzyme, Boehringer, Janssen, MSD, Celgene, Roche and Chugai, Martin Feuchtenberger Consultant of: Abbvie, BMS Chugai, Sanofi, Speakers bureau: Abbvie, BMS, Celgene, Chugai, Jansen-Cilag, Lilly, Pfizer, Roche, Sanofi, UCB, Georg Schett Speakers bureau: AbbVie, BMS, Celgene, Janssen, Eli Lilly, Novartis, Roche and UCB, Xenofon Baraliakos Grant/ research support from: Grant/research support from: AbbVie, BMS, Celgene, Chugai, Merck, Novartis, Pfizer, UCB and Werfen, Consultant of: AbbVie, BMS, Celgene, Chugai, Merck, Novartis, Pfizer, UCB and Werfen, Speakers bureau: AbbVie, BMS, Celgene, Chugai, Merck, Novartis, Pfizer, UCB and Werfen, Angela Zink Speakers bureau: AbbVie, Amgen, BMS, Gilead, Hexal, Janssen, 